\title{
A Novel Ultrasound-Based Registration for Image- Guided Laparoscopic Liver Ablation
}

\author{
Matteo Fusaglia MSc', Pascale Tinguely, MD², \\ Vanessa Banz, MD², Stefan Weber, PhD', and \\ Huanxiang Lu, PhD'
}

\begin{abstract}
Background. Patient-to-image registration is a core process of image-guided surgery (IGS) systems. We present a novel registration approach for application in laparoscopic liver surgery, which reconstructs in real time an intraoperative volume of the underlying intrahepatic vessels through an ultrasound (US) sweep process. Methods. An existing IGS system for an open liver procedure was adapted, with suitable instrument tracking for laparoscopic equipment. Registration accuracy was evaluated on a realistic phantom by computing the target registration error (TRE) for 5 intrahepatic tumors. The registration work flow was evaluated by computing the time required for performing the registration. Additionally, a scheme for intraoperative accuracy assessment by visual overlay of the US image with preoperative image data was evaluated. Results. The proposed registration method achieved an average TRE of $7.2 \mathrm{~mm}$ in the left lobe and $9.7 \mathrm{~mm}$ in the right lobe. The average time required for performing the registration was 12 minutes. A positive correlation was found between the intraoperative accuracy assessment and the obtained TREs. Conclusions. The registration accuracy of the proposed method is adequate for laparoscopic intrahepatic tumor targeting. The presented approach is feasible and fast and may, therefore, not be disruptive to the current surgical work flow.
\end{abstract}

\section{Keywords}

image guidance, laparoscopic ablation, liver, registration

\section{Introduction}

Surgical resection is considered the gold standard for the treatment of metastatic colorectal liver lesions, but $70 \%$ to $90 \%$ of the patients are not eligible for this procedure. $^{1,2}$ This high rate has encouraged the use of alternative surgical approaches such as microwave or radiofrequency tumor ablation. For ablation, the accurate placement of the needle's tip in the proximity of the tumor center has been shown to significantly reduce the recurrence rate. ${ }^{1}$

A laparoscopically assisted, percutaneous needlebased ablation procedure, which allows tumor ablation to be performed less invasively than open liver ablation procedures, has been proposed with the aid of 2D ultrasound (US) scanning. ${ }^{3}$ Compared with transcutaneous needle-based ablation, where a needle is inserted percutaneously through the aid of computed tomography (CT) imaging, this approach provides additional visual guidance in the form of laparoscopic imaging. The use of laparoscopic imaging enables an immediate control of the needle's trajectory, from the abdominal wall to the liver parenchyma, thus allowing a more intuitive needle insertion. Additionally, this approach eliminates the need of control scans-and, consequently, intraoperative radiation-which are required in interventional radiology to confirm that the

needle's trajectory is appropriate. Although laparoscopic-assisted ablation is more invasive than transcutaneous needle placement, it is less invasive than open-surgery liver ablations, thus providing the benefits related to laparoscopic surgery (ie, reduced scar size, trauma, and hospital stay).

During laparoscopic-assisted percutaneous needlebased ablation, the surgeon has to align the tumor's center and the needle within the US image. This alignment requires great concentration and in-depth experience, ${ }^{3}$ especially when tumors are poorly visible in the US image.

This disadvantage has been reduced by use employment of image-guided surgery (IGS) systems. ${ }^{4}$ Common IGS systems display the position of tracked surgical instruments relative to preoperative $3 \mathrm{D}$ virtual models of the organ (reconstructed from CT or magnetic

\footnotetext{
'University of Bern, Bern, Switzerland

${ }^{2}$ University Hospital of Bern, Bern, Switzerland

\section{Corresponding Author:}

Matteo Fusaglia, ARTORG Center for Biomedical Engineering Research, University of Bern, Murtenstrasse 50, 3010 Bern, Switzerland.

Email: matteo.fusaglia@artorg.unibe.ch
} 
Table I. Summary of Registration Methods That Use Ultrasound Imaging.

\begin{tabular}{|c|c|c|c|c|}
\hline Work & Registration Method & Accuracy & Application & $\begin{array}{l}\text { Applied } \\
\text { Registration }\end{array}$ \\
\hline Bao et al 6 & $\begin{array}{l}\text { Common landmarks between 3D } \\
\text { reconstruction and the phantom }\end{array}$ & $\begin{array}{l}\text { Localization error: } \\
5.3 \mathrm{~mm}\end{array}$ & $\begin{array}{l}\text { Laparoscopic } \\
\text { ablation/resection }\end{array}$ & Phantom \\
\hline Krücker et al ${ }^{9}$ & $\begin{array}{l}\text { Common landmarks between } \\
\text { preoperative 3D reconstruction and } \\
\text { intraoperative US imaging }\end{array}$ & $\begin{array}{l}\text { Root-mean-square (RMS) } \\
\text { registration error: } \\
\text { I.I } \mathrm{mm}\end{array}$ & Needle insertion & Phantom \\
\hline \multirow[t]{2}{*}{ Martens et al ${ }^{15}$} & $\begin{array}{l}\text { Two stages: } \\
\text { I. Coarse alignment: based on } \\
\text { landmark registration (acquired } \\
\text { with US probe) }\end{array}$ & $\begin{array}{l}\text { RMS error: 7.8-9.1 mm } \\
\text { (depending on } \\
\text { different magnetic } \\
\text { interferences) }\end{array}$ & Ablation/resection & $\begin{array}{l}\text { Phantom/planned } \\
\text { animal trial }\end{array}$ \\
\hline & 2. Fine registration: surface scan & & & \\
\hline
\end{tabular}

resonance imaging). These are then mapped with the available intraoperative data (intraoperative US, organ's position, CT imaging). This mapping, also called registration, determines a mathematical relationship from the preoperative $3 \mathrm{D}$ model coordinate system to the intraoperative image coordinate system. ${ }^{5,6}$ Obtaining an accurate registration represents a key aspect of the successful clinical use of IGS technologies in surgery.

Several research groups have reported the use of registration techniques that exploit the detection of superficial anatomical features of the liver (eg, skin fiducials, anatomical landmarks) through tracked instruments. $^{7-14}$ Although these methods provide accurate registration on the liver surface, they lack accuracy at the intraparenchymal structures (eg, tumors), thus hindering precise targeting of intrahepatic lesions.

Superior accuracy may be provided by intraoperative US because it visualizes intrahepatic structures that are closer to clinical targets. ${ }^{8}$ Registration techniques that exploit the detection of intraparenchymal hepatic structures (eg, vessel bifurcations, tumors) through US imaging have been reported in some studies. ${ }^{6,9,15}$ Through a calibrated and tracked laparoscopic ultrasound (LUS), anatomical landmarks that lie in the US image planes are manually identified and matched with the preoperative $3 \mathrm{D}$ model. A similar approach can also be found in 2 commercially available US systems (ACUSON S3000, HELX, Siemens Healthcare GmbH, Germany), where, after the identification of suitable landmarks, preoperative $3 \mathrm{D}$ reconstructions can be fused with the available US image. Although these systems provide advanced imaging visualization modules, they are designed for obstetrics and pediatrics applications and not for laparoscopic procedures. Table 1 provides a summary of the aforementioned registration methods.
Despite promising results being reported, the main disadvantage of these techniques is the time required to accurately define and detect the anatomical landmarks. ${ }^{16}$ This aspect may derive from the reduced spatial orientation and the lack of tactile feedback during laparoscopic procedures. We hypothesize that a promising approach to achieving an accurate and efficient registration is to use $3 \mathrm{D}$ reconstructions of the hepatic vasculature from LUS. By performing a LUS sweep over the intrahepatic region of interest (ROI; ie, in the proximity of tumors), an intraoperative 3D volume of the underlying vessels can be reconstructed. Subsequently, this intraoperative US-based 3D volume can be accurately registered to the preoperative $3 \mathrm{D}$ model. The registration accuracy, defined as target registration error (TRE), is expected to be $<10 \mathrm{~mm}$, which is commonly suggested as a safety margin. ${ }^{17}$ Additionally, we believe that because sweeping a LUS is more similar to intraoperative actions than defining landmarks, this technique would lead to a fast and intuitive work flow. Whereas previous works ${ }^{15,18,19}$ have reported the use of US-based 3D volumes, in the context of enhanced US guidance, to date, this technique has not been reported in the context of registration for laparoscopic IGS.

In this work, we present a novel registration method that reconstructs an intraoperative US-based $3 \mathrm{D}$ volume of the intrahepatic vessel anatomy and register it with the preoperative 3D model. To further ease the work flow, we propose a scheme for intraoperative accuracy assessment by visually inspecting the overlay of the preoperative 3D model on the intraoperative US. Our aim is to assess the registration accuracy by computing the TRE on a phantom with 5 intrahepatic target tumors, thus reflecting a clinically relevant scenario. Furthermore, the work flow efficiency is evaluated by analyzing the time required for performing the registration procedure and the relationship between the proposed intraoperative evaluation method and the obtained TREs. 


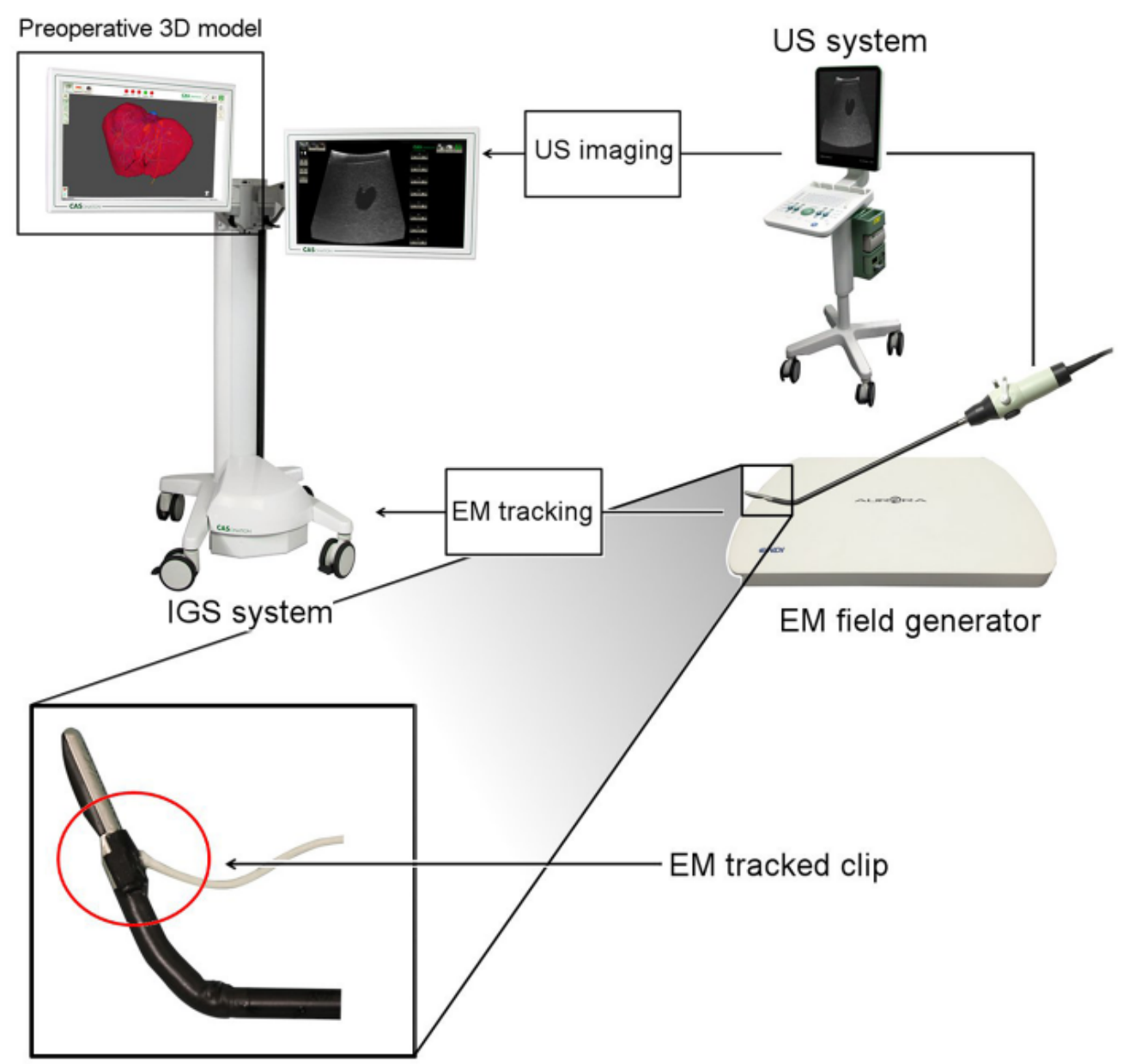

Figure I. System overview and functional components.

Abbreviations: US, ultrasound; EM, electromagnetic; IGS, image-guided surgery.

\section{Materials and Methods}

\section{System Overview}

A commercial IGS system for open liver procedures (CAScination AG, Switzerland) was supplemented with electromagnetic (EM) tracking (Aurora, Northern Digital, Canada) and an interface to a LUS (Figure 1; Flex800 with Probe 8666-RF, BK Medical, Denmark). The EM field generator creates a magnetic field that induces a current in the EM sensors and is subsequently translated in a $3 \mathrm{D}$ position. To measure the pose of the LUS image, an EM tracked clip was attached at the LUS tip and calibrated using the water bath method described in Prager et al. ${ }^{20}$ This LUS calibration determines the geometric transformation, which maps the US images in the IGS coordinate system, thus allowing the registration between the preoperative and intraoperative $3 \mathrm{D}$ US volumes.

The main components of the IGS system are the following:
1. Preoperative planning: The preoperative 3D model is displayed on a touch monitor, allowing the surgeon to selectively visualize structural and functional analysis (eg, portal vein territories, tumor volumetry).

2. Intraoperative imaging: The intraoperative US image is displayed on a second touch monitor and fed from an external US system (BK Medical, Denmark). An interactive interface allows the surgeon to manipulate the US parameters and modalities (eg, B-mode, Doppler, gain).

3. Registration: The registration work flow, which is the main focus of this work, is described in detail in the section 'Registration Work Flow'. It consists of 3 stages $^{21}$ :

\section{Selection of ROI}

2. Ultrasound sweep within the ROI

3. Computation of registration 


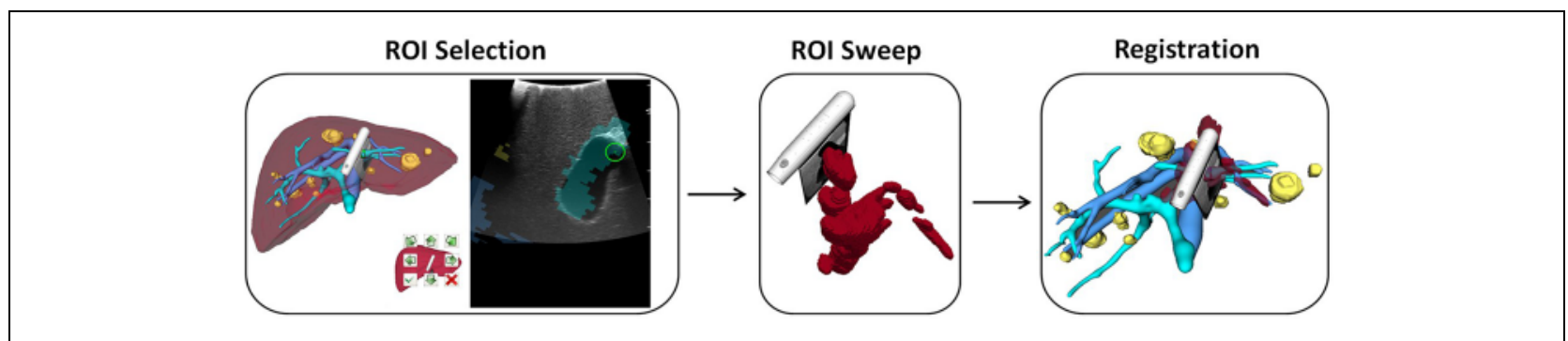

Figure 2. Registration work flow. ROI selection: 3D model and virtual LUS (left); simulated and real US image (right). ROI sweep: intraoperative vessel reconstruction (red). Registration: matching between the 3D model and the intraoperative vessel reconstruction.

Abbreviations: ROI, region of interest; LUS, laparoscopic ultrasound.

\section{Registration Work Flow}

Initially, a ROI is selected by defining a vessel bifurcation on the preoperative $3 \mathrm{D}$ model. Through this selection, the IGS system displays a virtual LUS on the preoperative $3 \mathrm{D}$ model and a simulated US image. This simulated US image depicts the structures that would be visible if the LUS is placed in the same position on the actual intraoperative liver. Based on this information, the surgeon sweeps the LUS over the liver until the structures depicted in the simulation correspond. This action provides a coarse alignment between the preoperative 3D model and the intraoperative US.

Second, the tracked LUS probe is swept within the ROI, the latter being delineated on the screen as a virtual cage. During this procedure, a real-time vessel segmentation based on difference of Gaussian ${ }^{22}$ reconstructs an intraoperative $3 \mathrm{D}$ volume of the vessels. The system depicts in real-time the LUS pose, providing the user with a visual feedback on where to move the probe within the ROI. As the system acquires US images, a progress bar indicates the level of task completion. Once a predefined amount of vessel points are detected, the $3 \mathrm{D}$ volume of the segmented vascular structures within the ROI is compounded.

Finally, the registration between the reconstructed US vasculature and the preoperative $3 \mathrm{D}$ model is computed. To account for the high imaging noise in the point set reconstructed from the US segmentation, a stochastic optimization approach based on generalized binary space partitioning tree is applied. ${ }^{23}$ The registration results in a mapping between the preoperative $3 \mathrm{D}$ model onto the intraoperative US 3D volume (Figure 2).

\section{Registration Quality Assessment}

After registration, the available mapping is applied to the visual context of the system allowing the user to view both the preoperative and the LUS image in a unified view (Figure 3). In our experiment, the user was asked to qualitatively examine the registration accuracy

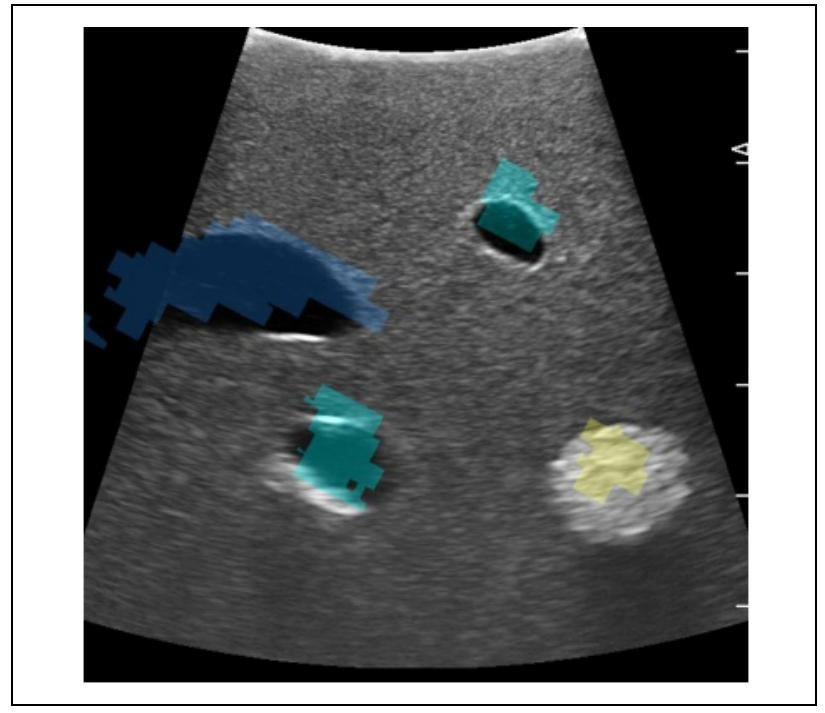

Figure 3. Preoperative 3D model (in color) rendered at the same perspective of the ultrasound image.

by inspecting the real-time alignment between the $3 \mathrm{D}$ model and the US image. If the registration result was not satisfying, the user was asked to redefine the ROI and/or to reacquire the US sweep until a satisfactory alignment was achieved. Additionally, a qualitative assessment of the registration accuracy in a range 1 to 10 (eg, $1=$ inaccurate to $10=$ accurate) was recorded by the system.

\section{Experimental Setup}

The aforementioned registration and work flow were evaluated on a multimodal liver phantom (IOUS, Japan) from which a preoperative 3D model was reconstructed from CT scans (MeVis Distant Services, Germany).

The liver phantom was positioned inside a laparoscopic trainer (Pharmabotics Ltd, UK), which was positioned on the surgical table. To simulate a real laparoscopic scenario, the trainer was inflated. The IGS system was positioned at 


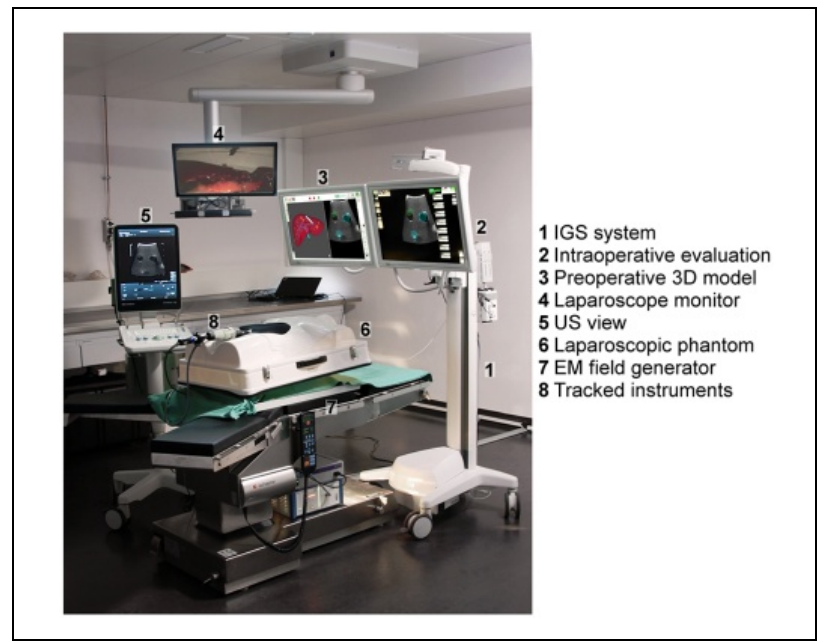

Figure 4. Experimental setup. Abbreviations: IGS, image-guided surgery; US, ultrasound; EM, electromagnetic.

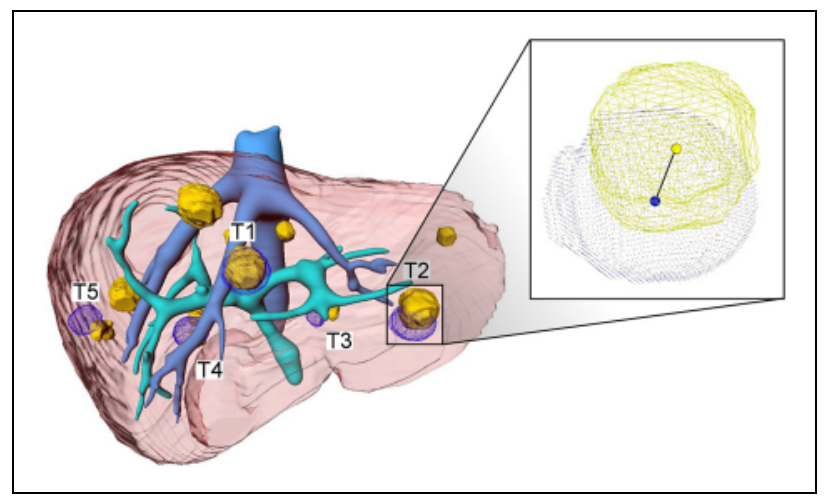

Figure 5. The evaluation of the registration is computed as the Euclidean distance between the registered tumors (yellow), and tumors reconstructed through US imaging (dotted blue).

the cranial end of the surgical table, with the monitors above the trainer. The EM field generator was positioned on the surgical table, below the trainer (Video $1, \quad$ available at http://sri.sagepub.com/supplemental). Surgical field visualization was accomplished by introducing a laparoscope (Storz, Germany) through a 10-mm trocar while the LUS was inserted directly through the trainer skin (Figure 4). The LUS probe was calibrated prior to the registration, and its accuracy was evaluated by visually assessing the corresponding position of an EM tracked pointer within the US image.

\section{Registration Accuracy}

In total, for both liver lobes, 30 registration attempts were performed by 3 individuals (ie, 10 attempts for each participant). Two participants were engineers, and 1 was a surgeon; all the participants were familiar with the concepts of IGS.
The registration procedure was repeated until a satisfactory level of accuracy was reached, as defined by the operating surgeon. To obtain a clinically relevant evaluation, we measured the registration accuracy at the intrahepatic tumor locations where the actual ablation would be performed during surgery. To that end, 5 underlying tumors ( 3 for the left lobe and 2 for the right lobe) were manually segmented from a 3D US scan and their centroid computed. The registration accuracy, defined as target registration error, was computed as the Euclidean distance between the registered tumor positions and those obtained through manual segmentation ${ }^{24}$ (Figure 5).

\section{Work Flow Efficiency}

For each registration attempt, the obtained TREs were then compared with the intraoperative qualitative assessments. Additionally, the time required for performing each registration step and the number of attempts needed to reach an acceptable registration accuracy were recorded.

\section{Results}

\section{Registration Accuracy}

The average TRE in the left lobe $(7.2 \mathrm{~mm})$ was lower than the one obtained in the right lobe $(9.7 \mathrm{~mm})$. Out of 5 targets, 4 presented a TRE $<10 \mathrm{~mm}$ (suggested as a safety margin in Mahnken and Ricke ${ }^{17}$ ). The target with a TRE above this threshold was positioned in segment VI.

A further experiment, which aimed to evaluate the spatial relationship between the TRE acquired during each successful attempt and the defined ROI, showed that the TRE increased relative to the distance to the ROI. By focusing on the TRE values inside the ROI, which is defined as a sphere of $50 \mathrm{~mm}$ around the acquired vessels, $76 \%$ of the values were $<10 \mathrm{~mm}$, whereas, by considering the entire TRE set, $86 \%$ of the TREs with value $<10 \mathrm{~mm}$ were inside the ROI (Table 2, Figure 6).

\section{Work Flow Efficiency}

The average number of registration attempts to obtain a successful registration in the right lobe was $4 \pm 1$, whereas this value decreased to $2.2 \pm 0.8$ in the left lobe. Figure 7 depicts the time required to perform the successful registrations. The average time required for performing the full process of a successful registration was 12 minutes. However, by considering only the registration and not the evaluation process, which was most relevant in our experiment, the average time for performing a successful registration was 7.4 minutes. By focusing on the individual steps during the registration 
Table 2. TREs for Each Target and Average Distance of the Targets to the Acquired ROI.

\begin{tabular}{lccccc}
\hline & & \multicolumn{2}{c}{ Right Lobe } \\
\hline & TI (Segment IV) & T2 (Segment III) & T3 (Segment IV) & T4 (Segment V) & T5 (Segment VI) \\
\hline TRE $(\mathrm{mm})$ & $7.8 \pm 2.4$ & $7.4 \pm 4.6$ & $6.3 \pm 2$ & $7.9 \pm 3.7$ & II.6 \\
Distance to ROI $(\mathrm{mm})$ & $42.8 \pm 4 . \mathrm{I}$ & $46.7 \pm 5.3$ & $21.9 \pm 4.5$ & $25 \pm 5.6$ & $50 \pm 14.8$ \\
\hline A &
\end{tabular}

Abbreviations: TRE, target registration error; ROI, region of interest.

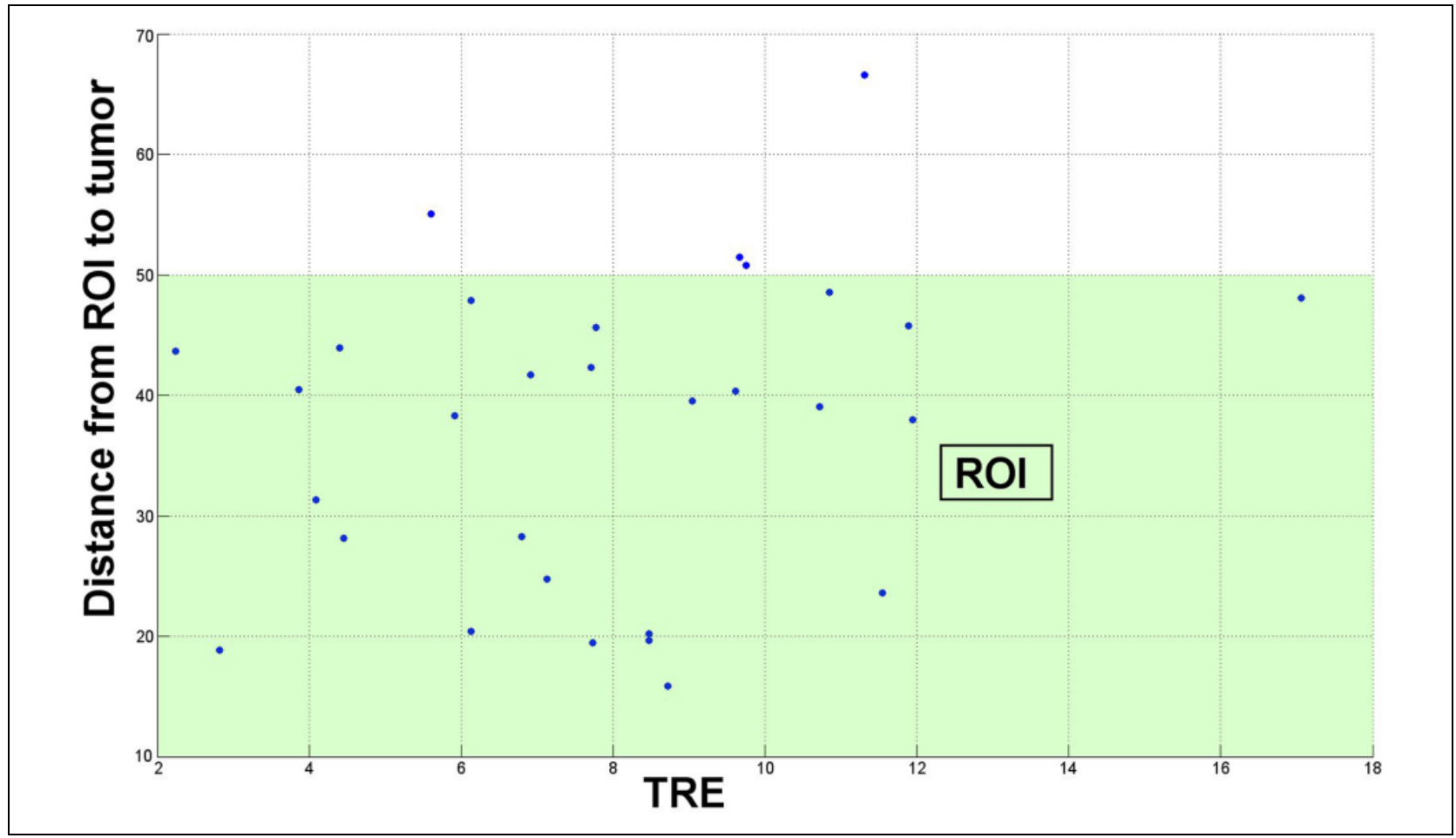

Figure 6. TRE of the successful registrations, as a function of distance of the targets to the ROI (depicted in green). Abbreviations: TRE, target registration error; ROI, region of interest.

process, the average time for ROI selection (4.9 minutes) and qualitative evaluation (4.5 minutes) were higher than for the ROI sweep (2.2 minutes) and registration ( 0.3 minutes). When considering the average time for ROI selection for each lobe, 6.7 minutes were needed for lesions located in the right lobe and 3.6 minutes for lesions in the left lobe. The time required for the ROI sweep varied depending on the number of vessels that were recognizable in the ROI.

\section{Registration Quality Assessment}

A positive correlation was found between the obtained TREs (mean $=20 ; \mathrm{SD}=13.3$ ) and the qualitative assessment of the registration accuracy (mean $=5.5 ; \mathrm{SD}$ $=2.6) ; r^{2}=0.75 ; \mathrm{n}=30$ (Figure 8$)$.

\section{Discussion}

IGS systems provide benefits for laparoscopic liver surgery by increasing the accuracy of tumor localization and allowing instrument guidance in complex surgical scenarios. In this work, we present a novel US-CT registration, which reconstructs an intraoperative USbased 3D volume of the underlying vessels in the context of laparoscopic IGS. Contrary to other registration techniques for laparoscopic procedures, the presented methodology relies mainly on an US sweeping process, which is intuitive and similar to common surgical actions. To enable a fast intraoperative registration procedure, we also propose a simple and intuitive surgical work flow, together with a direct verification scheme to assess the registration accuracy. This verification is accomplished by fusing in real time the intraoperative US image with the preoperative $3 \mathrm{D}$ models, rendered at the same perspective of the US image. To demonstrate the clinical applicability of the method, we set up the system in a near clinical scenario using a realistic liver phantom. Finally, a systematic evaluation of the registration accuracy and efficacy of the work flow, and a comparison between the proposed 


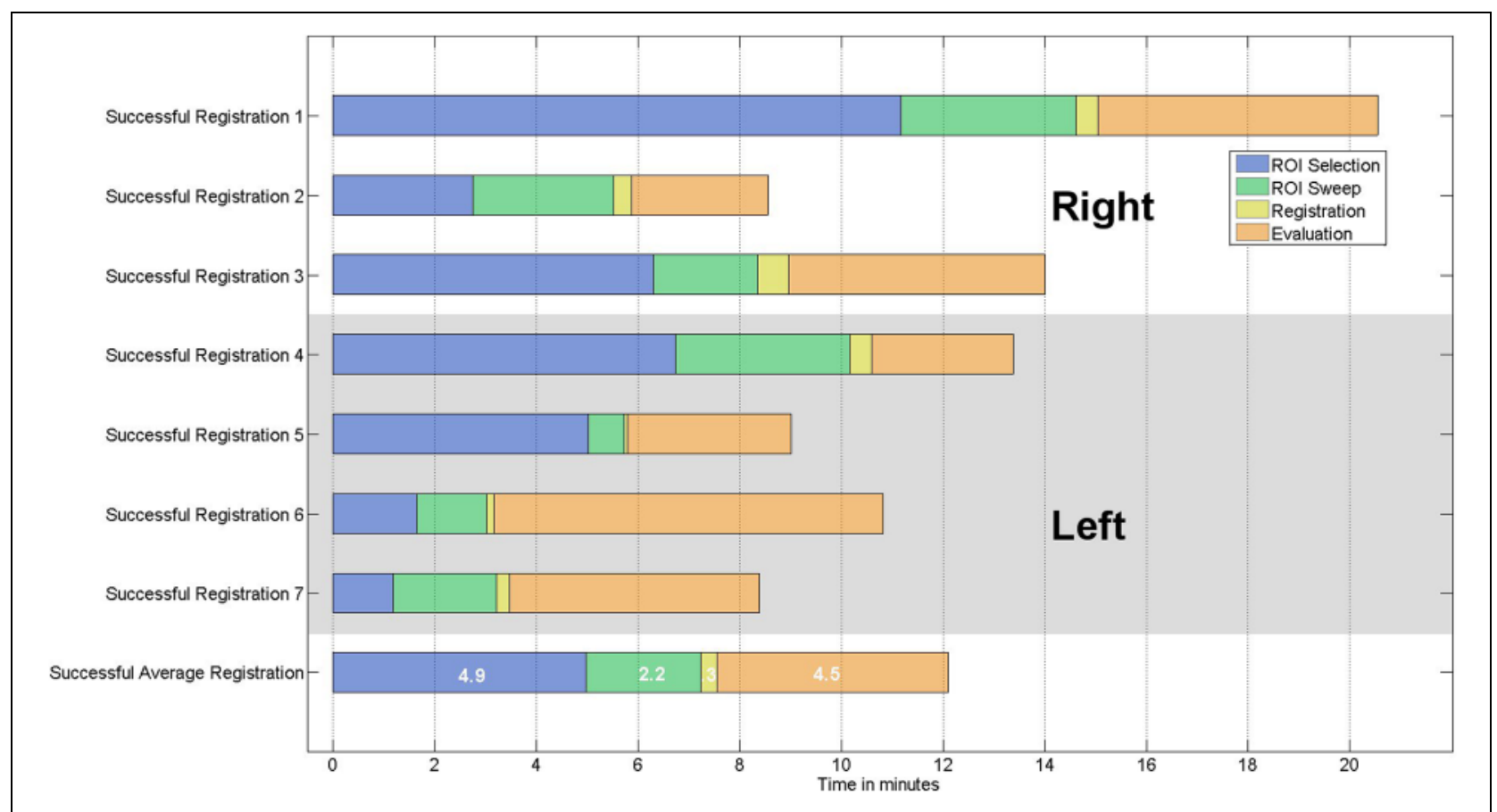

Figure 7. Time required for performing the successful registrations.

Abbreviation: ROI, region of interest.

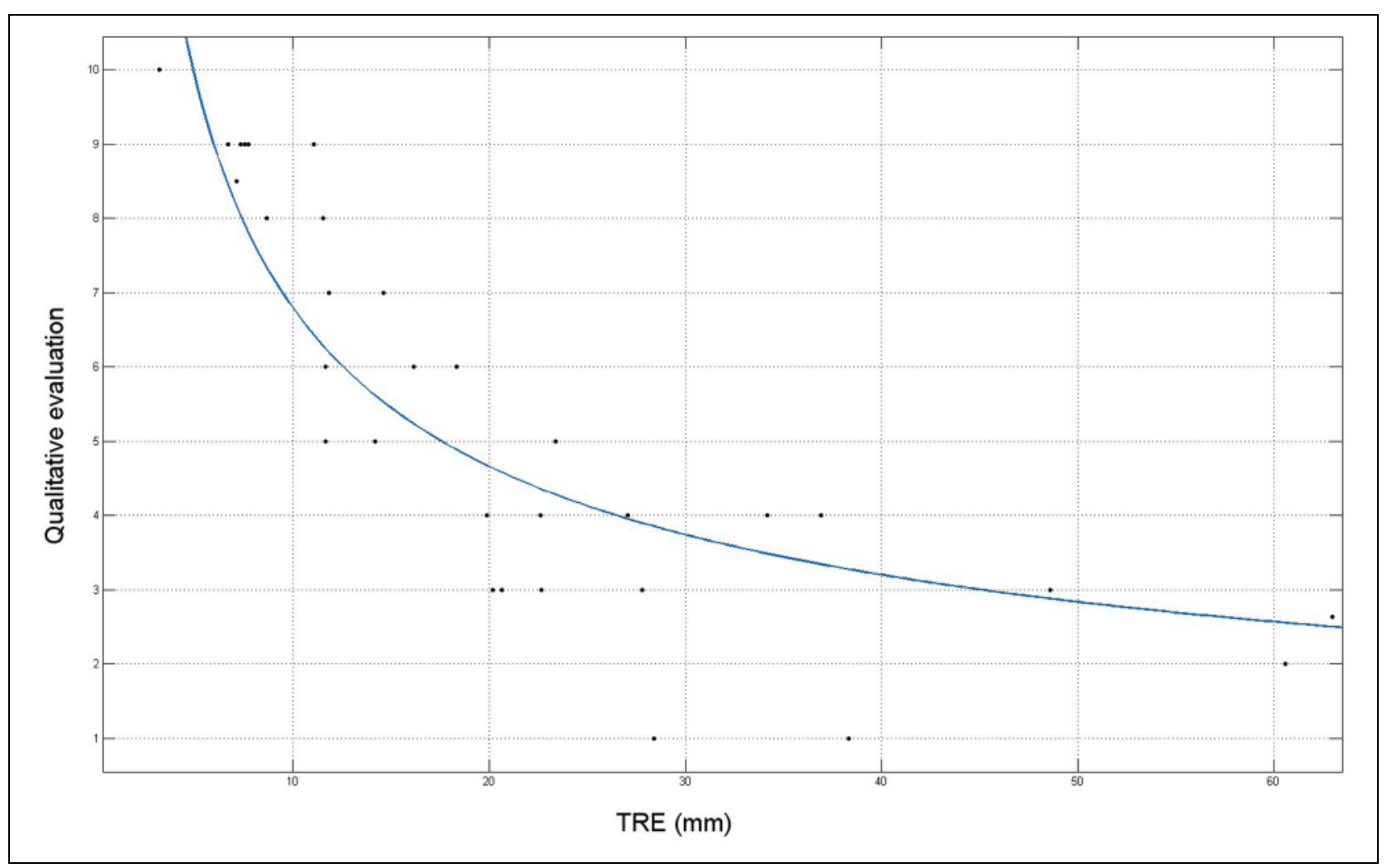

Figure 8. Correlation between the TRE and the qualitative evaluation. Abbreviation: TRE, target registration error. 
verification method and the registration accuracy are presented. The registration accuracy is evaluated by measuring the TRE of selected tumors, thus reflecting realistic errors obtained during laparoscopic ablation procedures.

Experimental results show a registration accuracy $<10 \mathrm{~mm}$ in both lobes, thus enabling its use in the clinical scenario, particularly for accurate ablation or biopsy procedures. We believe that these results will be similar to those obtained in the operating room because the targets were defined inside the liver and obtained through the tracked US, thus mimicking a realistic scenario. Despite these positive results, the registration accuracy in the right lobe was lower than the one found in the left lobe. This aspect can be attributed to a lower amount of recognizable anatomical features in the right lobe of the phantom, which led to a suboptimal vessel reconstruction and registration. This theory is validated by more time for the ROI definition and a higher time variance in the ROI sweep, which suggests a difficult recognition of clear vessel bifurcations. Another motivation can be phantom positioning and setup. Whereas the positioning of the phantom and the experimental setup resemble a realistic setup for left lobe operations, laparoscopic surgical techniques that operate in the right lobe are yet to be standardized. ${ }^{25,26}$ This implies that the adopted setup might not have been optimal for appropriate visualization and manipulation of the right lobe. A number of studies proposed a left lateral decubitus position, which allows more precise instrument handling. ${ }^{25,27}$

Additionally, we conduct an analysis evaluating the spatial relationship between the registration accuracy and the ROI. The results suggest that our method is accurate in the defined ROI. We believe that whereas optimizing the registration could improve the accuracy on the entire liver, it would result in a higher computational and operation time, leading to a more complex work flow. Conversely, while maintaining efficient work flow, our methodology allows high accuracy in a predefined region, which is relevant for the surgeon during a specific stage of the procedure. Additionally, this locality allows us to diminish the challenges related to tissue motion and deformation. ${ }^{28}$ In cases where the surgeon needs to change the ROI (eg, multiple tumor ablation), our work flow allows a fast registration in the new ROI. In this context, previous registrations could also be utilized as a coarse registration for the new ROI, thus facilitating identification and localization of the vessel within the new ROI.

The presented work flow allows one to achieve an accurate registration in $<15$ minutes. This result sustains the hypothesis that using conventional surgical actions (ie, LUS sweep) as registration means leads to a fast and intuitive work flow. Additionally, the positive correlation between the qualitative evaluation and the registration accuracy proved the efficacy of the verification method, based on overlaying preoperative 3D models on the intraoperative US image. This method allows a reliable intraoperative evaluation of the registration quality, which avoids a significant increase in the operating time.

Compared with previous studies, ${ }^{6,9,15}$ the presented system allows one to obtain a patient-to-image registration by defining a ROI. A ROI is larger than a set of landmarks and, therefore, easily identifiable, thus resulting in a faster and more intuitive registration process. Additionally, although the adoption of multimodal visualization techniques have been presented in previous studies, ${ }^{6,9,15}$ its use as an intraoperative validation scheme and the analysis of its efficacy represents a novelty. Finally, in contrast with previous studies, ${ }^{9,15}$ the registration accuracy, determined as TRE, represents a more clinically relevant measure because it measures the error at the target location through corresponding points, which were not used as registration means. $^{29}$

Despite the aforementioned positive results, the work flow efficiency in the right lobe presents several challenges. As mentioned before, the lack of clear anatomical features weakens the initialization procedure and consequently the entire registration process. The initialization procedure (eg, ROI selection) is also the most time-consuming procedure of the registration process. Both deficiencies can be alleviated by using a more robust US vessel reconstruction method, which will result in a finer and more detailed representation of the underlying anatomy. By achieving a finer representation of the underlying anatomy, the initialization procedure will be avoided, leading to shorter work flow times and an easier registration process. However, obtaining a more detailed vessel reconstruction would require higher computational time, resulting in a longer registration step. In future, the development of sophisticated US reconstruction methods, which exploit the use of different US imaging modalities (eg, US Doppler) will be investigated.

Another drawback is represented by the intraoperative liver respiratory motion and deformation that occurs as a result of organ manipulation with surgical instruments. Whereas these aspects are discarded in inanimate models, such as the one utilized in these experiments, future work will focus on the characterization of liver deformation. More specifically, in a clinical scenario, we plan to utilize mechanical high-frequency jet ventilation; this results in small tidal movements and a larger respiratory rate, which allows reduction in the cranio-caudal movements, constituting the predominant liver respiratory motion, ${ }^{30}$ from 20 to 5 mm. ${ }^{31,32}$ Additionally, a study performed by Zijlmans et $\mathrm{al}^{33}$ resulted in a modeling of the cranio-caudal movement, which can be described as a rigid transformation. These studies suggest that respiratory- 
related movements of the liver do not constitute the most critical part in the characterization of liver motion and deformation. On the other hand, challenges pertaining to liver deformation resulting from liver manipulation with surgical instruments can be compensated by the adoption of statistical models ${ }^{34}$ and nonrigid registrations. ${ }^{35-37}$

In conclusion, our study highlights the feasibility of a novel registration method in the context of laparoscopic IGS. The system presented eases the work flow and provides a reliable method to intraoperatively evaluate the registration accuracy; it could also be easily integrated into the clinical routine. Additionally, the validation method is versatile, enabling not only an intraoperative evaluation of the quality of the registration but also an enhanced US navigation view. Such a view could be used to locate vanishing lesions that are not visible through conventional US imaging. We believe that this work represents a significant step toward the use of navigation systems in the clinical routine.

\section{Author Contributions}

Study concept and design: All authors contributed equally to the study concept and design.

Acquisition of data: Matteo Fusaglia, Pascale Tinguely, and Huanxiang $\mathrm{Lu}$ are responsible for the data acquisition.

Analysis and interpretation: Matteo Fusaglia and Huanxiang $\mathrm{Lu}$ are responsible for data analysis and interpretation.

Study supervision: Matteo Fusaglia, Huanxiang Lu, and Stefan Weber supervised the study.

\section{Declaration of Conflicting Interests}

The author(s) declared the following potential conflicts of interest with respect to the research, authorship, and/or publication of this article: Matteo Fusaglia, Pascale Tinguely, Vanessa Banz, and Huanxiang Lu have no conflicts of interest or financial ties to disclose. Stefan Weber reports grants from Swiss Commission for Innovation and Technology and Eurostars Programme during the conduct of the study and is cofounder and shareholder of CAScination AG, a medical device manufacturer marketing navigation technology for minimally invasive liver surgery.

\section{Funding}

The author(s) received no financial support for the research, authorship, and/or publication of this article.

\section{References}

1. van Duijnhoven FH, Jansen MC, Junggeburt JM, et al. Factors influencing the local failure rate of radiofrequency ablation of colorectal liver metastases. Ann Surg Oncol. 2006;13:651-658.

2. Bentrem DJ, DeMatteo RP, Blumgart LH. Surgical therapy for metastatic disease to the liver. Annu Rev Med. 2005;56:139-156.

3. Sjølie E, Langø T, Ystgaard B, et al. 3D Ultrasoundbased navigation for radiofrequency thermal ablation in the treatment of liver malignancies. Surg Endosc. 2003; 17:933-938.

4. Unsgaard G, Ommedal S, Muller T, Gronningsaeter A, Nagelhus Hernes TA. Neuronavigation by intraoperative three-dimensional ultrasound: initial experience during brain tumor resection. Neurosurgery. 2002;50:804-812.

5. Hill DL, Batchelor P. Registration methodology: concepts and algorithms. In: Hajnal J, Hill D, Hawkes D. Medical Image Registration; CRC Press Taylor \& Francis Group. 2001:39-70.

6. Bao P, Warmath J, Galloway R Jr, Herline A. Ultrasound-tocomputer-tomography registration for image-guided laparoscopic liver surgery. Surg Endosc. 2005;19:424-429.

7. Marescaux J, Rubino F, Arenas M, Mutter D, Soler L. Augmented-reality-assisted laparoscopic adrenalectomy. JAMA. 2004;292:2211-2215.

8. Mårvik R, Langø $\mathrm{T}$, Tangen $\mathrm{GA}$, et al. Laparoscopic navigation pointer for three-dimensional image-guided surgery. Surg Endosc. 2004;18:1242-1248.

9. Krucker J, Xu S, Glossop N, Viswanathan A, Borgert J, Schulz H. An electro-magnetically tracked laparoscopic ultrasound for multi-modality minimally invasive surgery. In: International Congress Series. New York, NY: Elsevier; 2005:746-751.

10. Herline AJ, Herring JL, Stefansic JD, Chapman WC, Galloway RL Jr, Dawant BM. Surface registration for use in interactive, image-guided liver surgery. Comput Aided Surg. 2000;5:11-17.

11. Hammill CW, Clements LW, Stefansic JD, Wolf RF, Hansen PD, Gerber DA. Evaluation of a minimally invasive image-guided surgery system for hepatic ablation procedures. Surg Innov. 2014;21:419-426.

12. Feuerstein M, Mussack T, Heining SM, Navab N. Intraoperative laparoscope augmentation for port placement and resection planning in minimally invasive liver resection. IEEE Trans Med Imaging. 2008;27:355-369.

13. Rauth TP, Bao PQ, Galloway RL, et al. Laparoscopic surface scanning and subsurface targeting: implications for image-guided laparoscopic liver surgery. Surgery. 2007;142:207-214.

14. Shekhar R, Dandekar O, Bhat V, et al. Live augmented reality: a new visualization method for laparoscopic surgery using continuous volumetric computed tomography. Surg Endosc. 2010;24:1976-1985.

15. Martens V, Besirevic A, Shahin O and Kleemann M. LapAssistent-computer assisted laparoscopic liver surgery. In: Conference Proceedings of Biomedizinischen Technik (BMT). Rostock, Germany; 2010.

16. Langø $\mathrm{T}$, Tangen GA, Mårvik R, et al. Navigation in laparoscopy: prototype research platform for improved image-guided surgery. Minim Invasive Ther Allied Technol. 2008;17:17-33.

17. Mahnken AH, Ricke J. CT- and MR-Guided Interventions in Radiology. Vol 22. New York, NY: Springer; 2009.

18. Bao P, Sinha TK, Chen CCR, Warmath JR, Galloway RL, Herline AJ. A prototype ultrasound-guided laparoscopic radiofrequency ablation system. Surg Endosc. 2007;21:74-79.

19. Langø T, Vijayan S, Rethy A, et al. Navigated laparoscopic ultrasound in abdominal soft tissue surgery: technological overview and perspectives. Int J Comput Assist Radiol Surg. 2012;7:585-599.

20. Prager RW, Rohling RN, Gee AH, Berman L. Rapid calibration for 3-D freehand ultrasound. Ultrasound Med Biol. 1998;24:855-869. 
21. Ribes D, Peterhans $\mathrm{M}$, Anderegg $\mathrm{S}$ and Weber, S. Optimized workflow for US/MeVis-CT based registration in image guided liver surgery. In: Burgert $\mathrm{O}$, Schipper $\mathrm{J}$, Zachow S, eds. Deutsche Gesellschaft für Computer- und Roboterassistierte Chirurgie. Magdeburg, Germany: University of Magdeburg; 2013:55.

22. Dagon B, Baur C, Bettschart V. Real-time update of 3D deformable models for computer aided liver surgery. Paper presented at: 19th International Conference on Pattern Recognition; December 8-11, 2008; Tampa, FL.

23. Papazov C, Burschka D. Stochastic optimization for rigid point set registration. In: Boyle R, Parvin B, Koracin D. Advances in Visual Computing. New York, NY: Springer; 2009:1043-1054.

24. Fitzpatrick JM, West JB, Maurer CR Jr, Predicting error in rigid-body point-based registration. IEEE Trans Med Imaging. 1998; 17:694-702.

25. Belli G, Fantini C, D’Agostino A, et al. Laparoscopic segment VI liver resection using a left lateral decubitus position: a personal modified technique. $J$ Gastrointest Surg. 2008;12:2221-2226.

26. Goumard C, Farges O, Laurent A, et al; French Association for Hepatobiliary and Pancreatic Surgery. An update on laparoscopic liver resection: The French Hepato-Bilio-Pancreatic Surgery Association statement. $J$ Visc Surg. 2015;152:107-112.

27. Cherqui D, Husson E, Hammoud R, et al. Laparoscopic liver resections: a feasibility study in 30 patients. Ann Surg. 2000;232:753.

28. Heizmann O, Zidowitz S, Bourquain $\mathrm{H}$, et al. Assessment of intraoperative liver deformation during hepatic resection: prospective clinical study. World J Surg. 2010;34:1887-1893.

29. Fitzpatrick JM, West JB. The distribution of target registration error in rigid-body point-based registration. IEEE Trans Med Imaging. 2001;20:917-927.
30. Rohlfing T, Maurer CR Jr, O'Dell WG, Zhong J. Modeling liver motion and deformation during the respiratory cycle using intensity-based nonrigid registration of gated MR images. Med Phys. 2004;31:427-432.

31. Biro P, Spahn D, Pfammatter T. High-frequency jet ventilation for minimizing breathing-related liver motion during percutaneous radiofrequency ablation of multiple hepatic tumours. Br J Anaesth. 2009;102:650-653.

32. Warner M, Warner ME, Buck CF, Segura JW. Clinical efficacy of high frequency jet ventilation during extracorporeal shock wave lithotripsy of renal and ureteral calculi: a comparison with conventional mechanical ventilation. J Urol. 1988;139:486-487.

33. Zijlmans M, Langø T, Hofstad EF, Van Swol CF, Rethy A. Navigated laparoscopy-liver shift and deformation due to pneumoperitoneum in an animal model. Minim Invasive Ther Allied Technol. 2012;21:241-248.

34. Blackall JM, King AP, Penney GP, Adam A, Hawkes DJ. A statistical model of respiratory motion and deformation of the liver. In: Niessen WJ, Viergever MA. Medical Image Computing and Computer-Assisted InterventionMICCAI. New York, NY: Springer; 2001:1338-1340.

35. Schnabel JA, Tanner C, Castellano A, et al. A generic framework for non-rigid registration based on nonuniform multi-level free-form deformations. In: Niessen WJ, Viergever MA. Medical Image Computing and Computer-Assisted Intervention-MICCAI 2001, pp. 573581. New York, NY: Springer; 2001.

36. Masutani Y, Kimura F. Modally controlled free form deformation for non-rigid registration in image-guided liver surgery. In: Niessen WJ, Viergever MA. Medical Image Computing and Computer-Assisted InterventionMICCAI 2001, pp. 1275-1278. New York, NY: Springer; 2001.

37. Li H, Sumner RW, Pauly M. Global correspondence optimization for non-rigid registration of depth scans. In: Computer Graphics Forum, Vol. 27, No. 5, pp. 14211430. Chichester, UK: Wiley Online Library; 2008. 